\title{
CGC/saturation approach for soft interactions at high energy: a two channel model
}

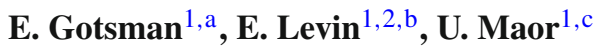 \\ ${ }^{1}$ Department of Particle Physics, School of Physics and Astronomy, Raymond and Beverly Sackler Faculty of Exact Science, Tel Aviv University, \\ Tel Aviv 69978, Israel \\ 2 Departemento de Física, Centro Científico-Tecnológico de Valparaíso, Universidad Técnica Federico Santa María, Avda. Espana 1680, \\ Casilla 110-V, Valparaíso, Chile
}

Received: 24 February 2015 / Accepted: 8 April 2015 / Published online: 30 April 2015

(C) The Author(s) 2015. This article is published with open access at Springerlink.com

\begin{abstract}
In this paper we continue the development of a model for strong interactions at high energy, based on two ingredients: the CGC/saturation approach and the BFKL Pomeron. In our approach, the unknown mechanism of confinement of quarks and gluons is characterized by several numerical parameters, which are extracted from the experimental data. We demonstrate that the two channel model successfully describes the experimental data, including both the value of the elastic slope and the energy behavior of the single diffraction cross section. We show that the disagreement with the experimental data of our previous single channel eikonal model (Gotsman et al., Eur Phys J C 75:1-18, 2015) stems from the simplified approach used for the hadron structure and is not related to our principal theoretical input, based on the $\mathrm{CGC} /$ saturation approach.
\end{abstract}

\section{Introduction}

In this paper we expand our approach of analyzing soft interactions at high energy based on two main ingredients: the Color Glass Condensate (CGC)/saturation effective theory for high energy QCD (see Ref. [1]) and references therein), and the BFKL Pomeron, which describes both soft and hard interactions at high energy [2-5]. The idea that there is only one BFKL Pomeron, which is not even a pole in the angular momentum plane (not a Reggeon; see Ref. [6] for details) presumes that the unknown mechanism of confinement in QCD of quarks and gluons is not important, and that its influence can be mimicked by the determination of several parameters of the CGC/saturation approach, which depend

\footnotetext{
ae-mail: gotsman@post.tau.ac.il

be-mails: leving@post.tau.ac.il; eugeny.levin@usm.cl

ce-mail: maor@post.tau.ac.il
}

on long distance physics. As an example of such a parameter, we mention the behavior of the scattering amplitude of the BFKL Pomeron at large impact parameters. This contradicts the hope that confinement would lead to a Pomeron, which is a Regge pole (see Ref. [7] and references therein). Unfortunately, due to the embryonic state of our understanding of confinement in QCD, we do not yet have a theoretical tool to differentiate between these two approaches. Hence, we concentrate our efforts on comparing the results of our approach, with relevant experimental data, hoping that an evaluation will allow us to check how viable our scenario is, and to find the specific features where our approach differs from one based on soft Pomeron calculus.

Our first attempt [8] shows that we can describe the main features of the data, but we found two results in our description which imply a potential problem for our approach: the result for the elastic slope is much smaller than the experimental measurement at the LHC energies, and the behavior of the cross section for single diffraction, which displays oscillating saturation as a function of energy. The goal of this paper is to show that these problematic features occur due to our oversimplified model in Ref. [8]. In this model, all shadowing corrections stem from two sources: the eikonal rescattering and the interaction of the BFKL Pomerons taken in the CGC/saturation approach. In this paper we develop a two channel model instead of the eikonal approximation, which is responsible for the low mass component in diffraction production, and which is an essential ingredient of all models of the high energy hadron scattering now on the market [9-20].

The paper is organized as follows. In the next section we describe the main theoretical input used in this paper. This section reviews the results that have been derived and employed in our previous paper [8]. In addition, we include a new formula for single diffraction dissociation, which is based on the closed form solution to the Balitsky-Kovchegov 
Fig. 1 MPSI approximation: the simplest diagram (a) and one Pomeron contribution (b). $C=\bar{\alpha}_{S}^{2} / 4 \pi \cdot \vec{x}_{1}-\vec{x}_{2}=\vec{r}^{\prime}$. $\frac{1}{2}\left(\vec{x}_{1}+\vec{x}_{2}\right)=\vec{b}^{\prime}$. The wavy lines describe BFKL Pomerons. The blobs stand for triple Pomeron vertices

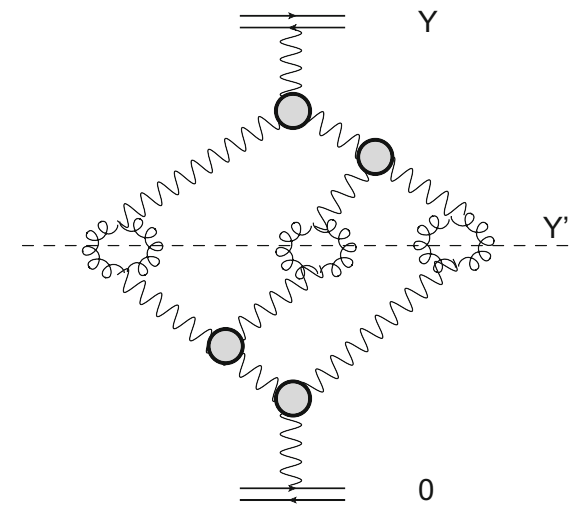

(a)

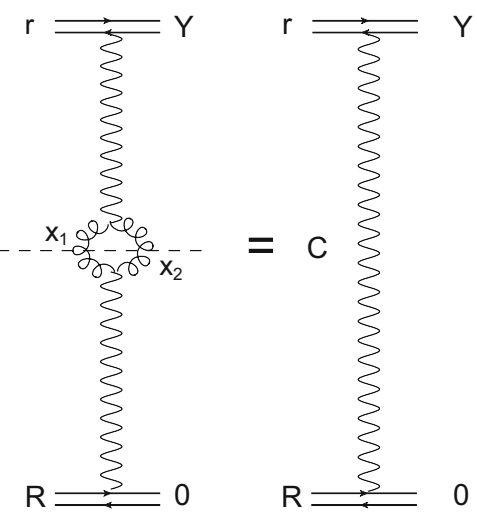

(b) equation [21]. In Sect. 3 we discuss the structure of our model and the phenomenological parameters that have been introduced. In this section, based on theoretical considerations, we estimate the range of the values of the parameters. The fourth section is devoted to the description and results of the fit.

\section{Theoretical input}

\subsection{Dressed Pomeron}

In the CGC/saturation approach (see Ref. [1] for the review of this approach), the scattering amplitude of two dipoles at high energy is described as the exchange of the dressed Pomeron, which can be calculated using the MPSI approximation [2226], displayed in Fig. 1. In Ref. [27-30] it was shown that the MPSI approximation is valid over a wide range of rapidities:

$Y \leq \frac{2}{\Delta_{\mathrm{BFKL}}} \ln \left(\frac{1}{\Delta_{\mathrm{BFKL}}^{2}}\right)$

From Fig. 1, it appears that the calculations can be performed in two stages: first, to find the sum of 'fan' diagrams for the rapidity regions $\left(0, Y^{\prime}\right)$ and $\left(Y^{\prime}, Y\right)$; and, second, to evaluate the diagram of Fig. 1a using the following sum rules for the BFKL Pomeron exchange [31,32] (see Fig. 1b for all notations):

$$
\begin{aligned}
& \frac{\alpha_{S}^{2}}{4 \pi} G_{\mathbb{P}}(Y-0, r, R ; b) \\
& \quad=\int \mathrm{d}^{2} r^{\prime} \mathrm{d}^{2} b^{\prime} G_{\mathbb{P}}\left(Y-Y^{\prime}, r, r^{\prime}, \vec{b}-\vec{b}^{\prime}\right) G_{\mathbb{P}}\left(Y^{\prime} r^{\prime}, R, \vec{b}-\vec{b}^{\prime}\right)
\end{aligned}
$$

In Eq. (2.1) $G_{\mathbb{P}}$ denotes the Green function of the BFKL Pomeron.

The first step can be accomplished by finding a solution to the Balitsky-Kovchegov (BK) non-linear equation [33-
35]. The solution has different forms in the three kinematic regions.

1. $r^{2} Q_{s}^{2}(Y, b) \ll 1$, where $Q_{s}$ denotes the saturation scale [31,44-48]. The non-linear corrections are small, and the solution is the BFKL Pomeron, which has the following form [2-5]:

$$
\begin{aligned}
& G_{\mathbb{P}}(Y, r, R ; b)=\left(w w^{*}\right)^{\frac{1}{2}} \sqrt{\frac{\pi}{4 D Y}} e^{\Delta_{\mathrm{BFKL}} Y-\frac{\ln ^{2} w w^{*}}{4 D Y}}, \\
& \text { with } \Delta_{\mathrm{BFKL}}=4 \ln 2 \bar{\alpha}_{S} \text { and } D=14 \zeta(3) \bar{\alpha}_{S} \\
& \quad=16.828 \bar{\alpha}_{S} .
\end{aligned}
$$

$G_{\mathbb{P}}(Y, r, R ; b)$ denotes the BFKL Pomeron Green function, $\bar{\alpha}_{S}$ the QCD coupling, $r$ and $R$ are the sizes of two interacting dipoles. $Y=\ln s$, where $s=W^{2}$. W denotes the energy of the interaction and $b$ the impact parameter of the scattering amplitude for two dipoles. We have

$w w^{*}=\frac{r^{2} R^{2}}{\left(\vec{b}-\frac{1}{2}(\vec{r}-\vec{R})\right)^{2}\left(\vec{b}+\frac{1}{2}(\vec{r}-\vec{R})\right)^{2}}$.

From Eq. (2.3) it is obvious that the BFKL Pomeron is not a pole in the angular momentum, but a branch cut, since its Y-dependence has an additional $\ln s$ term; it also does not reproduce the exponential decrease at large $b$, which follows from the general properties of analyticity and unitarity, for the exchange of the BFKL Pomeron $[49,50]$.

2. $r^{2} Q_{s}^{2}(Y, b) \sim 1$ (vicinity of the saturation scale). The scattering amplitude has the following form [51-53]:

$A \equiv G_{\mathbb{P}}(z)=\operatorname{Const}\left(r^{2} Q_{s}^{2}(Y, b)\right)^{1-\gamma_{c r}}$,

where Const denotes a constant, and where the critical anomalous dimension $\gamma_{c r}$, can be found from 


$$
\begin{aligned}
& \frac{\chi\left(\gamma_{c r}\right)}{1-\gamma_{c r}}=-\frac{\mathrm{d} \chi\left(\gamma_{c r}\right)}{\mathrm{d} \gamma_{c r}} \text { and } \\
& \chi(\gamma)=2 \psi(1)-\psi(\gamma)-\psi(1-\gamma) .
\end{aligned}
$$

3. $r^{2} Q_{s}^{2}(Y, b)>1$ (inside the saturation domain). The breakthrough that allows us to develop phenomenology based on the CGC/saturation approach was published in [54]. In this paper a simple approximation to the numerical solution of the BK equation was found, which is of the form

$$
N^{\mathrm{BK}}\left(G_{\mathbb{P}}(z)\right)=a\left(1-\exp \left(-G_{\mathbb{P}}(z)\right)\right)+(1-a) \frac{G_{\mathbb{P}}(z)}{1+G_{\mathbb{P}}(z)},
$$

with $a=0.65$ and $z=\ln \left(r^{2} Q_{s}^{2}(Y, b)\right)$ with $r=R$.

Using the solution of Eq. (2.7) we calculated the diagrams shown in Fig. 1a using Eq. (2.2) (see Refs. [8,54]). The result of this calculation gives the following expression for the Green function of the dressed Pomeron:

$$
\begin{aligned}
G_{\mathbb{P}}^{\text {dressed }} & \left(Y-Y_{0}, r, R, b\right) \\
= & a^{2}\left\{1-\exp \left(-T\left(Y-Y_{0}, r, R, b\right)\right)\right\} \\
& +2 a(1-a) \frac{T\left(Y-Y_{0}, r, R, b\right)}{1+T\left(Y-Y_{0}, r, R, b\right)} \\
& +(1-a)^{2}\left\{1-\exp \left(\frac{1}{T}\left(Y-Y_{0}, r, R, b\right)\right.\right. \\
& \left.\times \frac{1}{T\left(Y-Y_{0}, r, R, b\right)} \Gamma\left(0, \frac{1}{T\left(Y-Y_{0}, r, R, b\right)}\right)\right\},
\end{aligned}
$$

where $\Gamma(x)$ is the incomplete Euler gamma function (see 8.35 of Ref. [55]). The function $T\left(Y-Y_{0}, r, R, b\right)$ can be found from Eq. (2.2), and it has the form

$$
\begin{aligned}
& T\left(Y-Y_{0}, r, R, b\right)=\frac{\bar{\alpha}_{S}^{2}}{4 \pi} G_{\mathbb{P}}(z \rightarrow 0) \\
& \quad=\phi_{0}\left(r^{2} Q_{s}^{2}(R, Y, b)\right)^{1-\gamma_{c r}}=\phi_{0} S(b) e^{\lambda\left(1-\gamma_{c r}\right) Y},
\end{aligned}
$$

where we used two inputs: $r=R$ and $Q_{s}^{2}=\left(1 /\left(m^{2} R^{2}\right)\right)$ $S(m, b) \exp (\lambda Y)$. The parameter $\lambda$, in leading order of perturbative QCD, is given by $\lambda=\bar{\alpha}_{S} \chi\left(\gamma_{c r}\right) /\left(1-\gamma_{c r}\right)$. The parameter $m$ and the function $S(m, b)$ originate from nonperturbative QCD contributions, and they are parameterized as

$$
S(m b)=\frac{m^{2}}{\pi^{2}} \mathrm{e}^{-m b} \text { where } \int \mathrm{d}^{2} b S(b)=1 .
$$

$\phi_{0}$ can be calculated from the initial conditions using Eq. (2.3). Since we do not know these conditions, we will consider $\phi_{0}$ as an additional phenomenological parameter.

\subsection{Single diffraction}

The equation for the single diffraction production was proposed more than a decade ago [21]. In Ref. [21] it was shown that the equation has the same form as the BK equation [3343] for the function

$$
G\left(Y, Y_{0}, r, b\right)=2 N(Y, r, b)-N^{\mathrm{SD}}\left(Y, Y_{0}, r, b\right),
$$

where

$\sigma_{\text {diff }}\left(Y, Y_{0}, r\right)=\int \mathrm{d}^{2} b N^{\mathrm{SD}}\left(Y, Y_{0}, r, b\right)$

is the cross section for diffraction production, with the rapidity gap $\left(Y_{\text {gap }}=Y-Y_{M}\right)$ larger than $Y_{0}\left(Y_{\text {gap }} \geq Y_{0}\right)$.

$N(Y, r, b)$ is the imaginary part of the elastic amplitude; other notations are clarified in Fig. 2.

The difference between the elastic amplitude and $G\left(Y, Y_{0}\right.$, $r, b)$ is only in the initial condition, which for $G\left(Y, Y_{0}, r, b\right)$ is given by the following equation:

$G\left(Y, Y_{0}=Y, r, b\right)=2 N(Y, r, b)-N^{2}(Y, r, b)$,

where the last term denotes the elastic cross section.

Bearing in mind that the solution to Eq. (2.13) is given by Eq. (2.7), we can obtain a solution for single diffractive production. The cross section for the production of a bunch of hadrons with a mass from $M_{\min }$ to $M_{\max }$ can be written as $\sigma_{\text {diff }}\left(Y, Y_{\max }, Y_{\min }, r\right)=\int \mathrm{d}^{2} b \tilde{N}^{\mathrm{SD}}\left(Y, Y_{\max }, Y_{\min }, r ; b\right)$,

where the amplitude, $\tilde{N}^{\mathrm{SD}}\left(Y, Y_{\max }, Y_{\min } r ; b\right)$ takes the form

$$
\begin{aligned}
& \tilde{N}_{\mathrm{SD}}\left(Y, Y_{\max }, Y_{\min }, r ; b\right)=N^{B K}\left(T T\left(Y, Y_{\max }, b\right)\right) \\
& \quad-N^{B K}\left(T T\left(Y, Y_{\min }, b\right)\right),
\end{aligned}
$$

where $Y_{\max }=\ln \left(M_{\max }^{2} / s_{0}\right)$ and $Y_{\min }=\ln \left(M_{\min }^{2} / s_{0}\right)$.

For $T T\left(Y, Y_{M}, b\right)$ we have the following expression:

$$
\begin{aligned}
& T T\left(Y, Y_{M}, b\right)=\left(2 G^{\mathrm{dressed}}\left(T\left(Y-Y_{M}, b\right)\right)\right. \\
& \left.-\left(G^{\text {dressed }}\left(T\left(Y-Y_{M}, b\right)\right)\right)^{2}\right) e^{\left(1-\gamma_{c r}\right) \lambda\left(Y_{M}\right)} .
\end{aligned}
$$

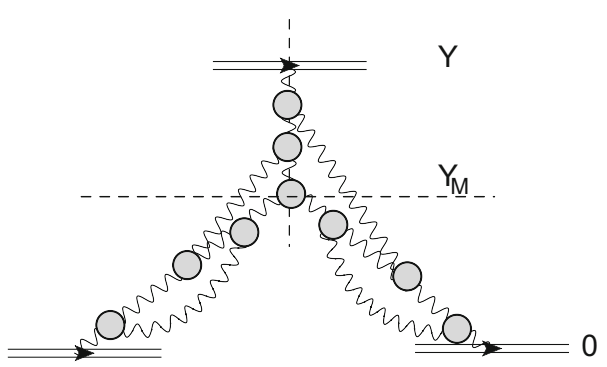

Fig. 2 MPSI approximation: the simplest diagrams for single diffraction production. The wavy lines describe BFKL Pomerons. The blobs stand for triple Pomeron vertices. The dotted line denotes the cut Pomeron. $Y_{M}=\ln \left(M^{2} / s_{0}\right)$, where $M$ is the mass of produced particles and $s_{0}$ is the scale taken to be of the order of $1 \mathrm{GeV}^{2}$ 


\subsection{Double diffraction}

We use $s$-channel unitarity to obtain the expression for the cross section for double diffractive production. Indeed, the unitarity constraints for the dressed Pomeron given by the diagrams of Fig. 1a take the form

$2 G^{\text {dressed }}(T(Y, b))=G^{\text {dressed }}(2 T(Y ; b))+N_{\mathrm{DD}}(Y ; b)$

where $G^{\text {dressed }}(2 T(Y ; b))$ describes all inelastic processes that are generated by the dressed Pomeron exchange. One can check this formula for the exchange of a single BFKL Pomeron. The general proof that the inelastic cross section due to the exchange of the dressed Pomeron is given by $G^{\text {dressed }}(2 T(Y ; b))$ can be found in Refs. [56-58].

From Eq. (2.17) the cross section of double diffractive production is equal to

$\sigma_{\mathrm{dd}}=\int \mathrm{d}^{2} b\left\{2 G^{\mathrm{dressed}}(T(Y, b))-G^{\mathrm{dressed}}(2 T(Y ; b))\right\}$.

In Eq. (2.18) we integrated over all possible masses without any restriction. We do not expect the cross section for double diffractive production of small masses to be large, and we believe that most of the contribution in this region of masses stem from the Good-Walker mechanism [59], which we consider in the next section.

\section{Main formulas and its phenomenological parameters}

\subsection{Two channel approximation}

In the previous section we reviewed the theoretical input from the $\mathrm{CGC} /$ saturation approach, used for calculating the Green function of the resulting Pomeron. In this section we discuss a model approach making two simplifications: one, that the eikonal formula for the hadron scattering amplitude satisfies $s$-channel unitarity; and two, that the simplified two channel model describes diffractive production in the low mass region. In this model, we replace the rich structure of the diffractively produced states by a single state, with wave function $\psi_{D}$. The observed physical hadronic and diffractive states can then be written

$\psi_{h}=\alpha \Psi_{1}+\beta \Psi_{2} ; \quad \psi_{D}=-\beta \Psi_{1}+\alpha \Psi_{2} ;$

where $\alpha^{2}+\beta^{2}=1$.

The functions $\psi_{1}$ and $\psi_{2}$ form a complete set of orthogonal functions $\left\{\psi_{i}\right\}$ which diagonalize the interaction matrix $\mathbf{T}$

$A_{i, k}^{i^{\prime} k^{\prime}}=<\psi_{i} \psi_{k}|\mathbf{T}| \psi_{i^{\prime}} \psi_{k^{\prime}}>=A_{i, k} \delta_{i, i^{\prime}} \delta_{k, k^{\prime}}$.
The unitarity constraints can be written as

$2 \operatorname{Im} A_{i, k}(s, b)=\left|A_{i, k}(s, b)\right|^{2}+G_{i, k}^{i n}(s, b)$,

where $G_{i, k}^{i n}$ denote the contributions of all non-diffractive inelastic processes, i.e. it is the summed probability for these final states to be produced in the scattering of a state $i$ off a state $k$. In Eq. (3.3) $\sqrt{s}=W$ is the energy of the colliding hadrons, and $b$ denotes the impact parameter. A simple solution to Eq. (3.3) at high energies has the eikonal form with an arbitrary opacity $\Omega_{i k}$, where the real part of the amplitude is much smaller than the imaginary part. We have

$A_{i, k}(s, b)=i\left(1-\exp \left(-\frac{\Omega_{i, k}(s, b)}{2}\right)\right) ;$
$G_{i, k}^{i n}(s, b)=1-\exp \left(-\Omega_{i, k}(s, b)\right)$.

Equation (3.5) implies that the probability that the initial projectiles $(i, k)$ will reach the final state interaction unchanged, regardless of the initial state re-scatterings, is given by $P_{i, k}^{S}=$ $\exp \left(-\Omega_{i, k}(s, b)\right)$.

The physical observables in this model can be written as follows:

elastic amplitude :

$$
a_{e l}(s, b)=i\left(\alpha^{4} A_{1,1}+2 \alpha^{2} \beta^{2} A_{1,2}+\beta^{4} A_{2,2}\right)
$$

elastic observables :

$$
\sigma_{\mathrm{tot}}=2 \int \mathrm{d}^{2} b a_{e l}(s, b) ; \quad \sigma_{\mathrm{el}}=\int \mathrm{d}^{2} b\left|a_{e l}(s, b)\right|^{2} ;
$$

optical theorem :

$$
\begin{aligned}
& 2 \operatorname{Im} A_{i, k}(s, t=0)=2 \int \mathrm{d}^{2} b \operatorname{Im} A_{i, k}(s, b) \\
& =\sigma_{e l}+\sigma_{\text {in }}=\sigma_{\text {tot }} ;
\end{aligned}
$$

single diffraction :

$$
\sigma_{\mathrm{sd}}^{\mathrm{GW}}=\int \mathrm{d}^{2} b\left(\alpha \beta\left\{-\alpha^{2} A_{1,1}+\left(\alpha^{2}-\beta^{2}\right) A_{1,2}+\beta^{2} A_{2,2}\right\}\right)^{2} ;
$$

double diffraction :

$$
\sigma_{\mathrm{dd}}^{\mathrm{GW}}=\int \mathrm{d}^{2} b \alpha^{4} \beta^{4}\left\{A_{1,1}-2 A_{1,2}+A_{2,2}\right\}^{2} .
$$

We denote by ' $G W$ ' the Good-Walker component, which is responsible for diffraction in the small mass region. This part of the diffraction production stems from the internal structure of the hadron, which we describe in a two channel model. It can be considered as diffraction in the region of the small mass, which is determined by the mass of the diffractive state characterized by $\psi_{D}$ in Eq. (3.1). Unfortunately, in the framework of our approach, the value of this mass is a new parameter of the model, and without introducing it, we 
cannot describe the distribution over mass in diffractive production. We would like to emphasize that in our approach, the production of a large mass is related to the structure of the dressed Pomeron, and these two components have quite different physics, and so cannot lead to double counting. Recall that high mass diffraction has been discussed in the one channel model [8], where the low mass diffraction is absent. The clear distinction between the two mechanisms can be illustrated in the constituent quark model for a hadron. The diffractive production in the region of small masses, which stems from the different states, can be described by the constituent quarks, while the high mass diffraction originates in quark-quark scattering.

In the eikonal approach we parametrize the arbitrary functions $\Omega_{i k}(s, b)$ in the form

$$
\begin{aligned}
& \Omega_{i k}(s, b) \\
& =\int \mathrm{d}^{2} b^{\prime} \mathrm{d}^{2} b^{\prime \prime} g_{i}\left(m_{i}, b^{\prime}\right) g_{k}\left(m_{k} b^{\prime \prime}\right) G^{\mathrm{dressed}}\left(T\left(Y, \vec{b}-\vec{b}^{\prime}-\vec{b}^{\prime \prime}\right)\right),
\end{aligned}
$$

where the vertex $g_{i}\left(m_{i}, b^{\prime}\right)$ is parameterized as

$$
\begin{aligned}
g_{i}\left(m_{i}, b^{\prime}\right)= & g_{i} S_{\mathbb{P}}\left(m_{i}, b^{\prime}\right), \quad \text { where } \\
& S_{\mathbb{P}}\left(m_{i}, b^{\prime}\right)=\frac{1}{4 \pi} m_{i}^{3} b^{\prime} K_{1}\left(m_{i} b^{\prime}\right) .
\end{aligned}
$$

$S_{\mathbb{P}}(b)$ in Eq. (3.11) is the Fourier transform of the form factor $1 /\left(1+q^{2} / m_{i}^{2}\right)^{2}$ and $K_{1}(z)$ denotes the modified Bessel function of the second kind (the McDonald function, see formulas 8.4 in Ref. [55]).

\subsection{Small phenomenological parameters and net diagrams}

The fits to experimental data (see Refs. $[8,20]$ and references therein) led to an unexpected result, viz.: the value of vertices for Pomeron-hadron interactions $\left(g_{i}\right)$, extracted from the fit of the data, turn out to be much larger than the vertex of triple Pomeron interaction $\left(G_{3 \mathbb{P}}\right.$; see Fig. 3).

Due to this small parameter $G_{3 \mathbb{P}} / g_{i} \ll 1$, we can improve the eikonal approximation, and sum a more general class of diagrams, the so-called net diagrams shown in Fig. 3. The analytical expression for the sum of the 'net' diagrams has

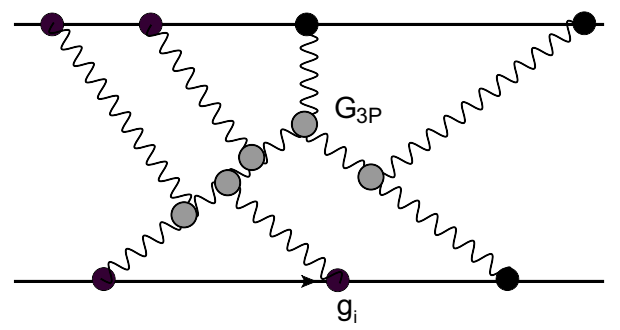

Fig. 3 The net diagram that gives the largest contribution if $g_{i} \gg$ $G_{3 \mathbb{P}}$. The wavy lines describe BFKL Pomerons. The blobs denote the triple Pomeron vertices been found (see Ref. [20]). Using the inequality observed in our previous paper [8], $m$ in Eq. (2.10) turns out to be much larger than both $m_{1}$ and $m_{2}$. Assuming this inequality to hold, we can simplify the integrals over $b^{\prime}$ and $b^{\prime \prime}$ in Eq. (3.10), re-writing this equation in the form

$$
\begin{aligned}
\Omega_{i k}(s, b)= & \int \mathrm{d}^{2} b^{\prime} \mathrm{d}^{2} b^{\prime \prime} g_{i}\left(m_{i}, b^{\prime}\right) g_{k}\left(m_{k} b^{\prime \prime}\right) G^{\text {dressed }} \\
& \times\left(T\left(Y, \vec{b}-\vec{b}^{\prime}-\vec{b}^{\prime \prime}\right)\right) \\
= & \left(\int \mathrm{d}^{2} b^{\prime \prime} G^{\mathrm{dressed}}\left(T\left(Y, b^{\prime \prime}\right)\right)\right) \\
& \times \int \mathrm{d}^{2} b^{\prime} g_{i}\left(m_{i}, b^{\prime}\right) g_{k}\left(m_{k}, \vec{b}-\vec{b}^{\prime}\right) .
\end{aligned}
$$

The summation of the 'net' diagram is then given by the following simplified expression (see Ref. [20] for details):

$$
\begin{aligned}
& \Omega(Y ; b) \\
& =\int \mathrm{d}^{2} b^{\prime} \frac{g_{i}\left(\vec{b}^{\prime}\right) g_{k}\left(\vec{b}-\vec{b}^{\prime}\right) \widetilde{G}^{\mathrm{dressed}}(T)}{1+1.29 \widetilde{G}^{\mathrm{dressed}}(T)\left[g_{i}\left(\vec{b}^{\prime}\right)+g_{k}\left(\vec{b}-\vec{b}^{\prime}\right)\right]},
\end{aligned}
$$

where

$\widetilde{G}^{\text {dressed }}(T)=\int \mathrm{d}^{2} b G^{\mathrm{dressed}}(T(Y, b))$.

The coefficient 1.29 results from the extraction of the value of $G_{3 \mathbb{P}}$ from the CGC/saturation approach, which has been considered in the previous section.

\subsection{Diffraction production in the region of large mass}

In this section we also include, in the process of diffraction production, the mechanism of production that originates from the dressed Pomeron, and that has been discussed in Sect. 2 .

For single diffraction the large mass contribution can be written

$$
\begin{aligned}
& \sigma_{\text {sd }}^{\text {large mass }}=2 \int \mathrm{d}^{2} b\left\{\alpha^{6} A_{1 ; 1,1}^{\mathrm{sd}} e^{-2 \Omega_{1,1}^{D}(Y ; b)}\right. \\
& \quad+\alpha^{2} \beta^{4} A_{1 ; 2,2}^{\mathrm{sd}} e^{-2 \Omega_{1,2}^{D}(Y ; b)} \\
& \quad+2 \alpha^{4} \beta^{2} A_{1 ; 1,2}^{\mathrm{sd}} e^{-\left(\Omega_{1,1}^{D}(Y ; b)+\Omega_{1,2}^{D}(Y ; b)\right)} \\
& \quad+\beta^{2} \alpha^{4} A_{2 ; 1,1}^{\mathrm{sd}} e^{-2 \Omega_{1,2}^{D}(Y ; b)} \\
& \quad+2 \beta^{4} \alpha^{2} A_{2 ; 1,2}^{\mathrm{sd}} e^{-\left(\Omega_{1,2}^{D}(Y ; b)+\Omega_{2,2}^{D}(Y ; b)\right)} \\
& \left.\quad+\beta^{6} A_{2 ; 2,2}^{\mathrm{sd}} e^{-2 \Omega_{2,2}(Y ; b)}\right\}
\end{aligned}
$$

where

$$
\begin{aligned}
& \Omega_{i, k}^{D}(Y ; b) \\
& =\int \mathrm{d}^{2} b^{\prime} \frac{g_{i}\left(\vec{b}^{\prime}\right) g_{k}\left(\vec{b}-\vec{b}^{\prime}\right) \bar{G}^{\text {dressed }}(T)}{\left(1+1.29 \bar{G}^{\text {dressed }}(T)\left[g_{i}\left(\vec{b}^{\prime}\right)+g_{k}\left(\vec{b}-\vec{b}^{\prime}\right)\right]\right)^{2}},
\end{aligned}
$$




$$
\begin{aligned}
& A_{i ; k, l}^{\mathrm{sd}}\left(Y, Y_{\max }, Y_{\min } ; b\right) \\
& \quad=\int \mathrm{d}^{2} b^{\prime} \sigma_{\mathrm{diff}}\left(Y, Y_{\max }, Y_{\min }, 1 / m\right) g_{i} g_{k} g_{l} S_{\mathbb{P}}\left(b^{\prime}, m_{i}\right) \\
& S_{\mathbb{P}}\left(\vec{b}-\vec{b}^{\prime}, m_{k}\right) S_{\mathbb{P}}\left(\vec{b}-\vec{b}^{\prime}, m_{l}\right),
\end{aligned}
$$

where $\sigma_{\text {diff }}\left(Y, Y_{\max }, Y_{\min }, r\right)$ is given by Eq. (2.12), $Y=$ $\ln \left(s / s_{0}\right), Y_{\max }=\ln \left(M_{\max }^{2} / s_{0}\right)$ and $Y_{\min }=\ln \left(M_{\min }^{2} / s_{0}\right)$. $M_{\max }$ and $M_{\min }$ are the largest and smallest mass produced in the diffractive processes.

Equation (3.15) has a simple physical meaning: each term is the product of probability to produce a mass diffractively from the dressed Pomeron (term $\left.\exp \left(-\sum \Omega\right)\right)$, and the probability of the process of single diffraction, from the dressed Pomeron $\left(A_{i ; k, l}\right)$.

For the double diffraction production at large mass we have

$$
\begin{aligned}
& \sigma_{\mathrm{dd}}^{\text {large mass }}=\int \mathrm{d}^{2} b\left\{\alpha^{4} A_{1,1}^{\mathrm{dd}} e^{-2 \Omega_{1,1}^{D}(Y ; b)}\right. \\
& \left.\quad+2 \alpha^{2} \beta^{2} A_{1,2}^{\mathrm{dd}} e^{-2 \Omega_{1,2}^{D}(Y ; b)}+\beta^{4} A_{2,2}^{\mathrm{dd}} e^{-2 \Omega_{2,2}^{D}(Y ; b)}\right\},
\end{aligned}
$$

$$
\begin{aligned}
& A_{i, k}^{\mathrm{dd}} \\
& \quad=\int \mathrm{d}^{2} b g_{i} g_{k} S_{\mathrm{DD}}^{i, k}(b) \sigma_{\mathrm{dd}}(Y) \text { where } S_{\mathrm{DD}}^{i, k}(b) \\
& \quad=\int \mathrm{d}^{2} b^{\prime} S_{p}\left(b^{\prime}, m_{i}\right) S_{p}\left(\vec{b}-\vec{b}^{\prime}, m_{k}\right),
\end{aligned}
$$

where $\sigma_{\mathrm{dd}}$ is given by Eq. (2.18).

\subsection{Estimates for the values of the phenomenological parameters}

We have two sets of phenomenological parameters, which need to be determined by fitting to the experimental data. The first set is related to the description of the dressed Pomeron: $\phi_{0}, \lambda$, and $m$. The parameter $\phi_{0}$, in principle, could be found from the solution of the BFKL equation at low energy. Unfortunately, we do not know the initial condition for BFKL evolution. This is the reason why we extract this parameter by fitting to the experimental data. The value of $\phi_{0}$ should be of the order of $\alpha_{S}$, and therefore, we expect that this parameter will be small. $\lambda$ determines the energy dependence of the saturation scale. We know the theoretical value of $\lambda=2 \bar{\alpha}_{S}\left(\psi(1)-\psi\left(\frac{1}{2}\right)\right) /\left(1-\gamma_{c r}\right)$ where $\psi(z)$ denotes the Euler psi-function (see formulas 8.36 of Ref. [55]) and $\gamma_{c r}=0.37$ (see Ref. [1]). On the other hand, the value of $\lambda \approx 0.3$ has been extracted from DIS and nucleus-nucleus scattering for the energy dependence of the saturation scale [60-65]. The mass $m$ plays a twofold role: it determines the impact parameter dependence, and it gives the size of the typical dipole in a hadron. This is a dimensional parameter, whose value is determined by nonperturbative QCD, and, at the moment, we have no theoretical input for this quantity. In all our formulas, we use the intuitive assumption that the mass $m$, is the largest mass in our model.

The second set of parameters: $g_{i}$ and $m_{i}$ as well as values of $\alpha$, are associated with the description of the wave functions in our two channel model. This set is of non-perturbative origin, and it has to be determined by fitting to the data. In our formulas we assume that $g_{i} \gg G_{3 \mathbb{P}}$ and $m \gg m_{i}$. This assumption is based on our past experience with soft Pomeron models for high energy scattering [20].

\section{The result of the fit}

\subsection{Cross sections}

We have eight parameters to be determined by fitting to the experimental data on total, inelastic and elastic cross sections, single and double diffractive production cross sections, and the slope of the forward elastic differential cross section. The value of the minimal energy for data that we use is $W=$ $0.546 \mathrm{TeV}$ as, starting from this energy, the CGC/saturation approach is able to describe the data on inclusive production in proton-proton collisions (see Ref. [60-63]). For lower energies, saturation occurs in ion-ion and proton-ion collisions, but not in proton-proton collisions [64].

The quality of the fit can be judged from Fig. 4. The fitted parameters are tabulated in Table 1.

From Table 1 we see that the qualitative features of the two channel model are similar to those of the one channel model [8]. The values of all the parameters have the same hierarchy i.e. $g_{i} \gg G_{3 \mathbb{P}}\left(G_{3 \mathbb{P}}=1.29 \mathrm{GeV}^{-1}\right.$ in our approach $)$ and $m \gg m_{i}$. We recall that this hierarchy is necessary for our formulas to be valid. $\lambda \approx 0.3$ follows from the estimates of the energy dependence of the saturation momentum from DIS and ion-ion scattering experiments. $\phi_{0}$ is small, as is expected from QCD. It is worthwhile mentioning, using estimates from leading order perturbative QCD, that this value of $\lambda$ leads to a very small value of the QCD coupling: $\bar{\alpha}_{S} \approx 0.07$. This is a general problem for the CGC/saturation approach, and we assign the difference between the phenomenological value of $\lambda$ and its leading order estimates to higher order corrections, which give a large contribution to this observable $[66,67]$.

It should be noted that both in Fig. 4 and in Table 2 we calculate the high mass diffraction component with the additional restriction $\Delta \eta \geq 3$.

We obtain a good description of the data, including the energy behavior of the elastic slope and the single diffraction cross section. Therefore, we have elucidated the problems that occurred in the one channel model [8]. We show in 
Fig. 4 Comparison with the experimental data: the energy behavior of the total (a), inelastic (b), elastic cross sections (c), as well as the elastic slope $\left(B_{e l}, \mathbf{d}\right)$ and single diffraction (e) and double diffraction (f) cross sections. The solid lines show our present fit. The data has been taken from Ref. [76] for energies less than the LHC energy. At the LHC energy for total and elastic cross section we use TOTEM data [72-74] and for single and double diffraction cross sections are taken from Ref. $[68,69]$. The dotted line in $\mathbf{f}$ is based on Eq. (4.1) (see text following this equation) (a)

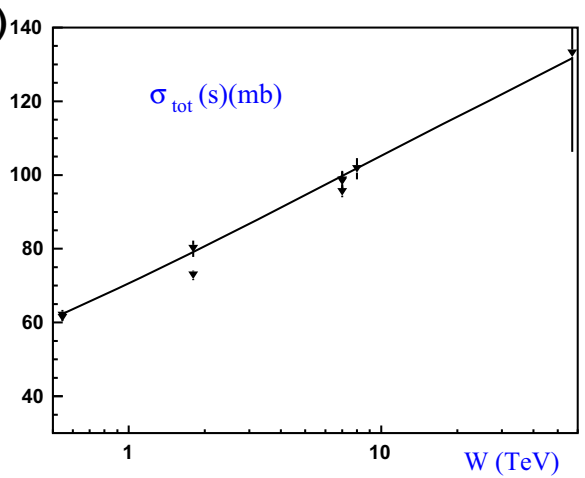

(c)

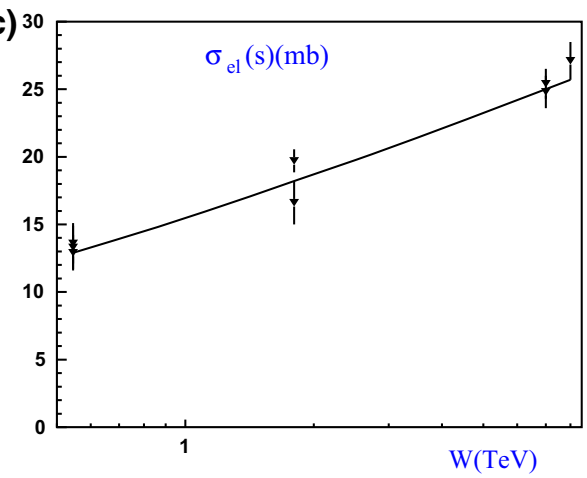

(e)

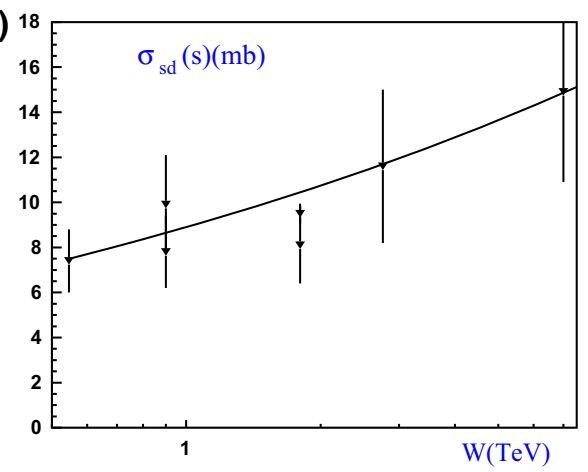

(b) 1

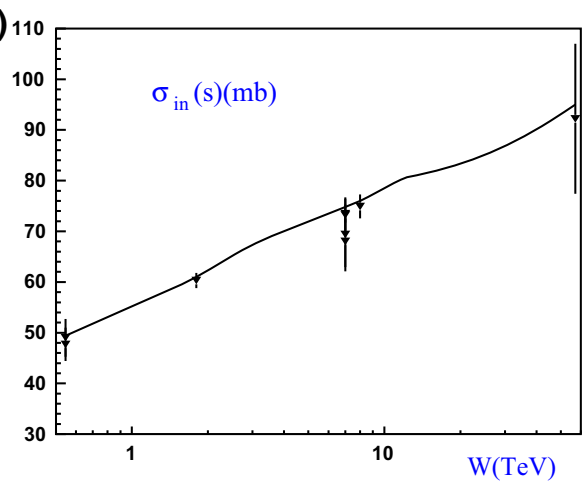

(d)

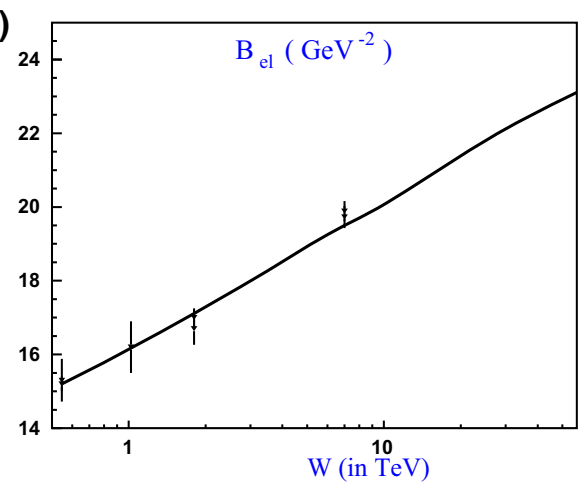

(f)

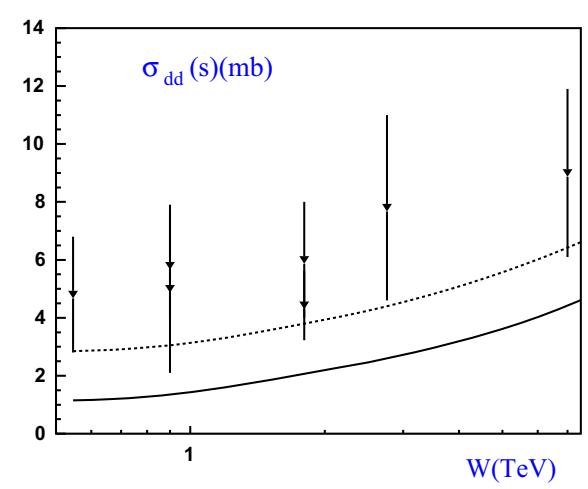

Table 1 Fitted parameters of the model

\begin{tabular}{lllllllll}
\hline Model & $\lambda$ & $\phi_{0}$ & $g_{1}\left(\mathrm{GeV}^{-1}\right)$ & $g_{2}\left(\mathrm{GeV}^{-1}\right)$ & $m(\mathrm{GeV})$ & $m_{1}(\mathrm{GeV})$ & $m_{2}(\mathrm{GeV})$ & $\beta$ \\
\hline 2 channel & 0.38 & 0.0019 & 110.2 & 11.2 & 5.25 & 0.92 & 1.9 & 0.58 \\
1 channel & 0.323 & 0.019 & 25.7 & n/a & 6.35 & 0.813 & n/a & n/a \\
\hline
\end{tabular}

this paper that the oscillatory behavior of the single diffraction and the weak energy dependence of the elastic slope were artifacts of the oversimplified one channel model, and they are not associated with our main theoretical input: the CGC/saturation approach.

A deficiency in our two channel model is the description of the double diffractive cross section, as can be seen in Fig. 4 . This failure is due to the small Good-Walker contribution for the production of low masses. It predicts a cross section of about $1 \mathrm{mb}$. To understand the sources of this small value, we note that Eq. (3.10) leads to the cross section for double diffraction, which can be calculated using the factorization relation (see Ref. [18] for example). We have

$\sigma_{\mathrm{dd}}^{\mathrm{GW}}=\frac{\left(\sigma_{\mathrm{sd}}^{\mathrm{GW}} B_{\mathrm{sd}}\right)^{2}}{\left(\sigma_{\mathrm{el}} B_{\mathrm{el}}\right) B_{\mathrm{dd}}}$,

To obtain an estimate we assume that $B_{\mathrm{sd}} \approx B_{\mathrm{el}} \approx B_{\mathrm{sd}}$, where $B$ denotes the slopes of the various cross sections; using our estimates for the elastic and single diffractive cross sections, we obtain $\sigma_{\text {dd }}^{\mathrm{GW}} \sim 3 \mathrm{mb}$ at $\mathrm{W}=7 \mathrm{TeV}$. 
If we evaluate the cross section of Good-Walker component using Eq. (4.1) we obtain a reasonable description of the experimental data (see dotted curve in Fig. 4f). We wish to stress that the shadowing corrections that follow from the CGC/saturation approach do not violate the factorization properties of Eq. (4.1). The source of the violation of Eq. (4.1) is the net diagrams, which in our model incorporate the interactions of the dressed Pomerons. To illustrate the influence of this interaction, we calculated the slopes at $\mathrm{W}=$ $7 \mathrm{TeV}$. We obtain $B_{\mathrm{el}}=19.45 \mathrm{GeV}^{-2} ; B_{\mathrm{sd}}=25.08 \mathrm{GeV}^{-2}$, and $B_{\mathrm{dd}}=44 \mathrm{GeV}^{-2}$. Using Eq. (4.1) with these values decreases the estimated $\sigma_{\mathrm{dd}}$ by a factor of almost 3. Hence, the main effect of the dressed Pomerons interactions is to increase the typical $b$, in the impact parameter distribution for double diffractive cross sections.

On the other hand, the small cross section for double diffraction arises naturally in other theoretical approaches [18], and the cross section, measured by the TOTEM collaboration [75] in the restricted region of the produced mass is rather small $\left(\sigma_{\mathrm{dd}}=116 \pm 25 \mu \mathrm{b}\right.$ for $\Delta Y_{M}=1.8$ and $\left.\left|Y-Y_{\max }\right|=4.7\right)$. Bearing this in mind, we are not too disappointed with our description of the double diffractive cross section.

In Table 2 we present the results of our model for the various cross sections at different energies. Note that for single and double diffraction, the large mass region is responsible for substantial contributions (more than half of the cross section). These contributions originate from the structure of the dressed Pomeron, and they are the main theoretical input from the CGC/saturation approach.

As has been mentioned, what we call "low mass" is the contribution coming from the Good-Walker sector, while "high mass" is the contribution which stems from the dressed Pomeron diagrams. Unfortunately, we are not able to put a precise $M_{X}$ bound on the two contributions, since we do not know the typical mass of the Good-Walker component (the state described by $\psi_{D}$ in Eq. (3.1) in our approach. We have to bear this deficiency in mind if we want to discuss the detailed data for diffraction production coming from TOTEM collaboration [72-75]. As is seen from Table 2, in our model the cross section of the low mass single dissociation at $7 \mathrm{TeV}$ is equal to $8.7 \mathrm{mb}$, while the TOTEM [72-74] estimate is $2.6 \pm 2.2 \mathrm{mb}$ for $M_{X}<3.4 \mathrm{GeV}$. As we have mentioned we cannot consider our GW component as being responsible for this mass range. On the other hand, the TOTEM estimate is not a direct measurement but is obtained from their Monte Carlo. There are other Monte Carlo estimates by Ostapshenko [16] who finds $\sigma_{\mathrm{sd}}=3.9 \mathrm{mb}$ for $M_{X}<3.4 \mathrm{GeV}$, and by Pogosyhan [14] who estimates $\sigma_{\mathrm{sd}}=4 \mathrm{mb}$ for the same mass range. The only conclusion that we can derive from this discussion is that the GW typical mass is larger than $3.4 \mathrm{GeV}$.

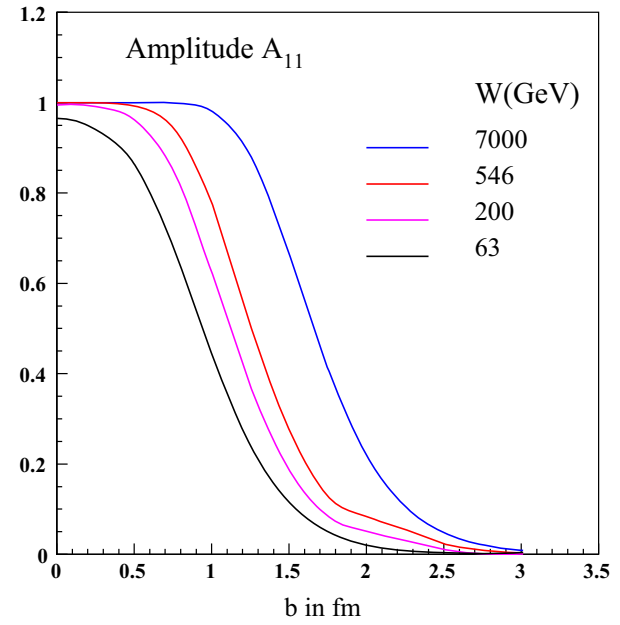

Fig. 5 The impact parameter dependence of the 'black' $A_{1,1}$ amplitude, as a function of energy $W$

We should also mention that our estimation is in agreement with the ALICE result of $\sigma_{\mathrm{sd}}=14.9_{-5.9}^{+3.4} \mathrm{mb}$ for $0<M_{X}^{2}<$ $0.5 s$ at $\mathrm{W}=7 \mathrm{TeV}$.

\subsection{Amplitudes}

It is instructive to compare the two models which are both based on CGC/saturation approach: the one channel model developed in Ref. [8] and the two channel model of this paper. In Fig. 6 we plot the amplitudes $A_{i k}$ for the two channel model and elastic amplitude for the one channel model, at different energies, as functions of the impact parameter, $b$. From Fig. 6a we note that one of our amplitudes $\left(A_{2,2}\right.$ is rather small at $7 \mathrm{TeV}$, while the amplitude $A_{11}$ reaches the unitarity limit for $b \leq 1.5 \mathrm{fm}$. It is interesting to compare this behavior with the results of two channel model, based on the Pomeron as a Regge pole [20]. One notes a drastic difference in the dependence on the impact parameter, as well as the relative values of the amplitude. Qualitatively, the Pomeron interaction leads to stronger shadowing corrections than the CGC/saturation approach.

We would like to draw the reader's attention to the energy behavior of the 'black' $A_{1,1}$ amplitude, as shown in Fig. 5. We note that the size of the black part of the amplitude for our model is much smaller than for the Pomeron-based model, discussed in [20]. The second observation is that this amplitude becomes transparent at low energies. One of the unpleasant features of the Pomeron (Regge pole) models is the fact that the black component already appears at very low energies. The present model does not have this deficiency, providing a smooth transition from a transparent to the black disc picture. 
Table 2 The values of cross sections versus energy. $\sigma_{\mathrm{sd}}^{L M}$ and $\sigma_{\mathrm{dd}}^{L M}$ denote the cross sections for diffraction dissociation in the low mass region, for single and double diffraction, which stem from the Good-
Walker mechanism. $\sigma_{\mathrm{sd}}^{H M}$ and $\sigma_{\mathrm{dd}}^{L M}$ are used for diffraction in high mass, coming from the dressed Pomeron contributions

\begin{tabular}{|c|c|c|c|c|c|c|c|}
\hline \multirow[t]{2}{*}{$W(\mathrm{TeV})$} & \multirow[t]{2}{*}{$\sigma_{\text {tot }}(\mathrm{mb})$} & \multirow[t]{2}{*}{$\sigma_{\mathrm{el}}(\mathrm{mb})$} & \multirow[t]{2}{*}{$B_{\mathrm{el}}\left(\mathrm{GeV}^{-2}\right)$} & \multicolumn{2}{|c|}{ Single diffraction } & \multicolumn{2}{|c|}{ Double diffraction } \\
\hline & & & & $\sigma_{\mathrm{sd}}^{L M}(\mathrm{mb})$ & $\sigma_{\mathrm{sd}}^{\mathrm{HM}}(\mathrm{mb})$ & $\sigma_{\mathrm{dd}}^{\mathrm{LM}}(\mathrm{mb})$ & $\sigma_{\mathrm{dd}}^{\mathrm{HM}}(\mathrm{mb})$ \\
\hline $0 . .576$ & 62.3 & 12.9 & 15.2 & 5.64 & 1.85 & 0.7 & 0.46 \\
\hline 0.9 & 69.2 & 15 & 16 & 6.25 & 2.39 & 0.77 & 0.67 \\
\hline 1.8 & 79.2 & 18.2 & 17.1 & 7.1 & 3.35 & 0.89 & 1.17 \\
\hline 2.74 & 85.5 & 20.2 & 17.8 & 7.6 & 4.07 & 0.97 & 1.62 \\
\hline 7 & 99.8 & 25 & 19.5 & 8.7 & 6.2 & 1.15 & 3.27 \\
\hline 8 & 101.8 & 25.7 & 19.7 & 8.82 & 6.55 & 1.17 & 3.63 \\
\hline 13 & 109.3 & 28.3 & 20.6 & 9.36 & 8.08 & 1.27 & 5.11 \\
\hline 14 & 110.5 & 28.7 & 20.7 & 9.44 & 8.34 & 1.27 & 5.4 \\
\hline 57 & 131.7 & 36.2 & 23.1 & 10.85 & 15.02 & 1.56 & 13.7 \\
\hline
\end{tabular}

Fig. 6 Amplitudes for one and two channel model versus $b$ at different energies. a The amplitude $A_{i k}(b)$ at $\mathrm{W}=7 \mathrm{TeV}$ as a function of the impact parameter $b$ in our model. The same amplitudes appear in $\mathbf{b}$ for the two channel model based on the Pomeron interaction [20].

The energy behavior of the elastic amplitude is plotted in $\mathbf{a}$, c for the one and two channels models, respectively. The figure for the one channel model is taken from Ref. [8]
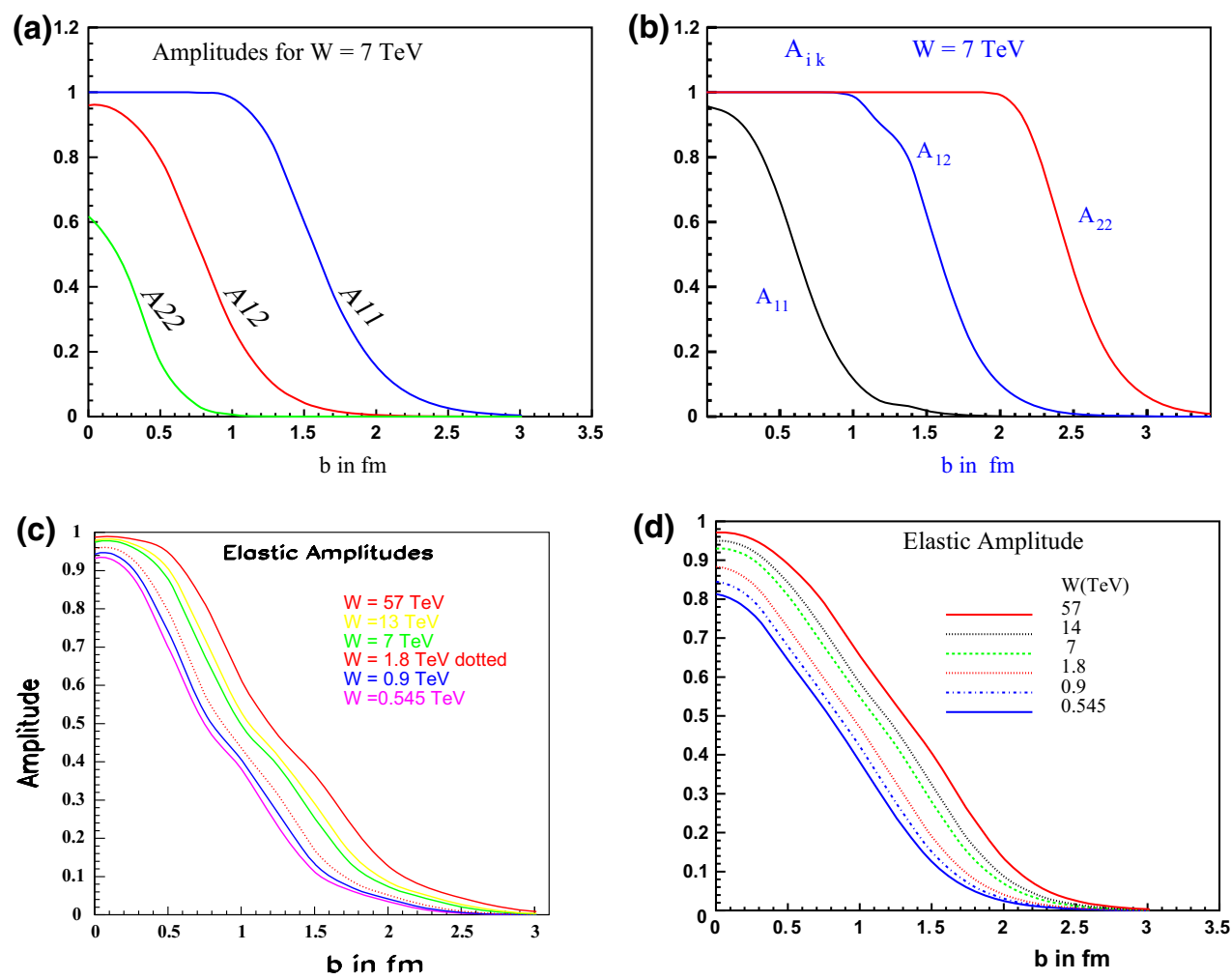

(d)

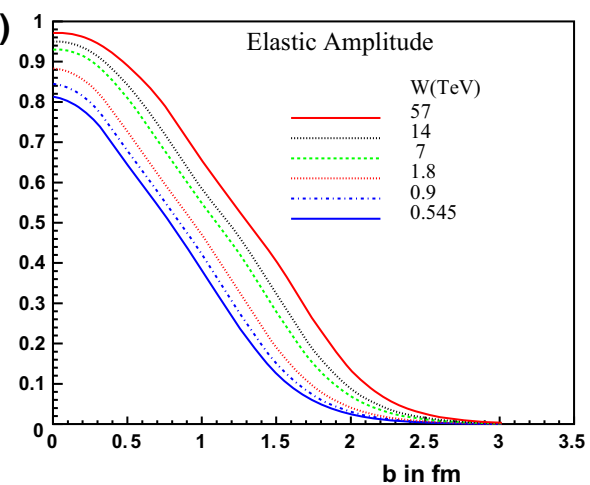

Comparing Fig. 6c, d we conclude that the two channel model leads to weaker shadowing corrections than the one channel model.

Looking at Fig. 6, it is difficult to avoid the pessimistic conclusion that until we know the non-perturbative QCD structure of the hadrons, the high energy interaction of hadrons cannot be treated in a unique fashion, but is doomed to be sensitive to ad hoc assumptions.

This statement can be illustrated by Fig. 7 where we plot the $\mathrm{d} \sigma / \mathrm{d} b^{2}$ for the diffraction production processes. The low mass diffraction has pronounced peripheral features, having minima at $b=0$ and at $b=1 \div 1.5 \mathrm{fm}$. However, for high mass diffraction the main contribution stems from $b=0$. Single diffraction in the region of high mass does not have a minimum at $b=0$, while in double diffraction such a minimum is seen, but it is very shallow. Comparing these distributions with the one channel model, we see that for single diffraction, the pattern of the impact parameter distribution is similar in both cases. Double diffraction has a very clear peripheral distribution in the one channel model, but in the two channel model, the double diffraction dependence on $b$ is similar to the single diffraction. We believe that this 
Fig. $7 \mathrm{~d} \sigma / \mathrm{d} b^{2}$ versus $b$ for different processes of diffraction production and different energies. ' $\mathrm{hm}$ ' ('lm') and 'sd' ('dd') denote diffraction in high mass (low mass) region and single (double) diffraction, respectively. Figures $\mathbf{e}, \mathbf{f}$ are taken from our paper on the one channel model based on the CGC/saturation approach [8]. a High mass single diffraction, b low mass single diffraction, c high mass double diffraction, d low mass double diffraction, e single diffraction ( $1 \mathrm{ch}$. model) and $\mathbf{f}$ double diffraction (1ch. model) (a)

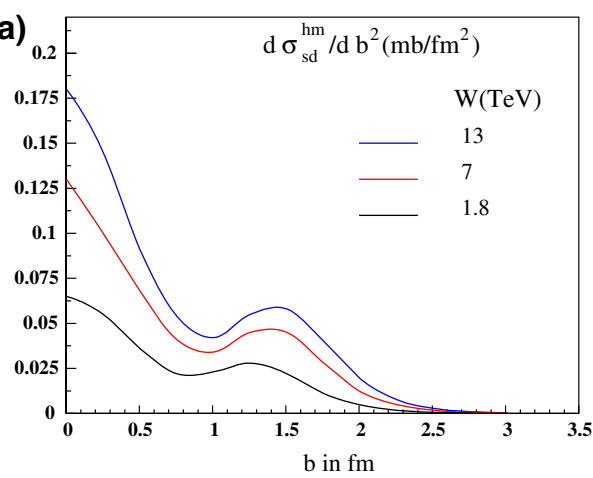

(c)

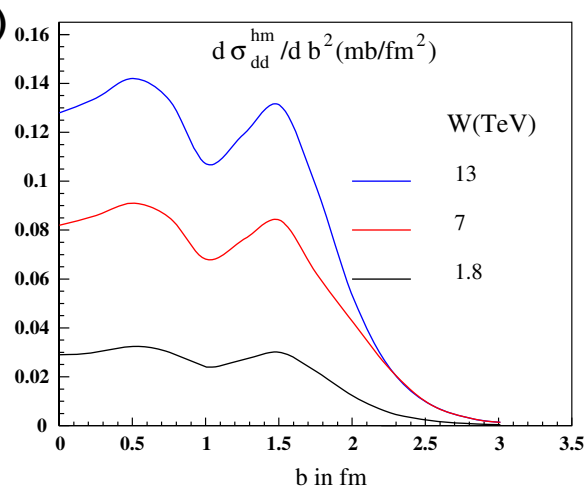

(e)

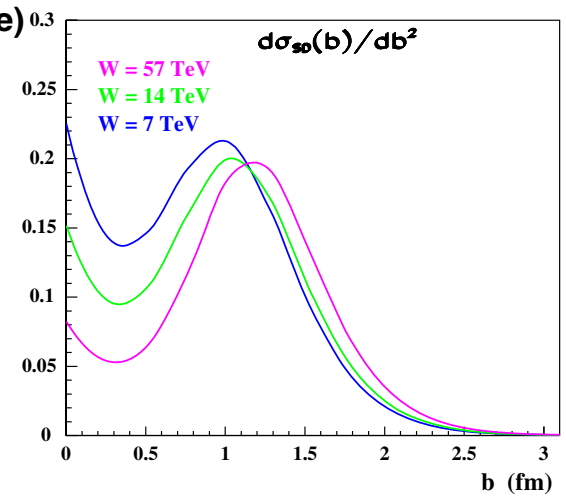

(b)

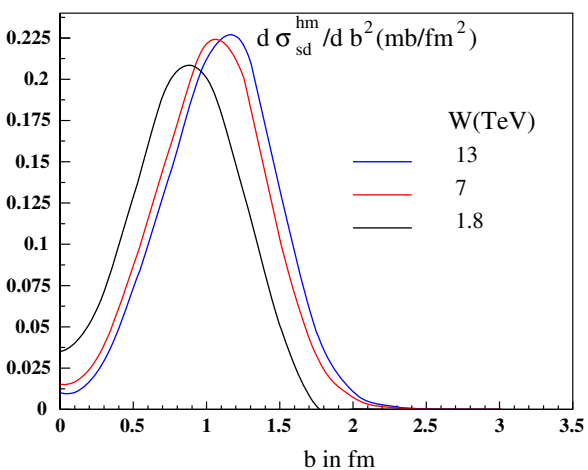

(d)

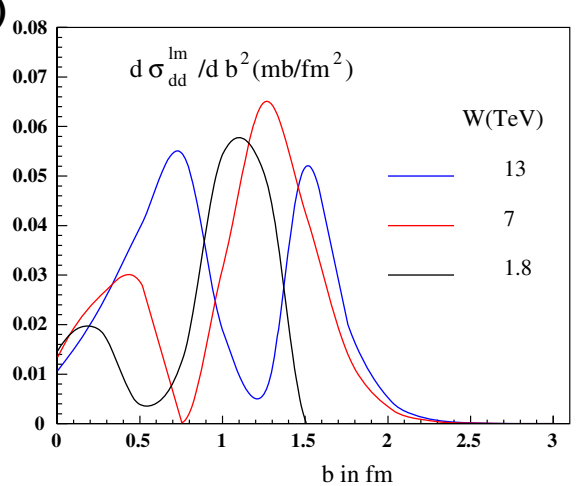

(f)

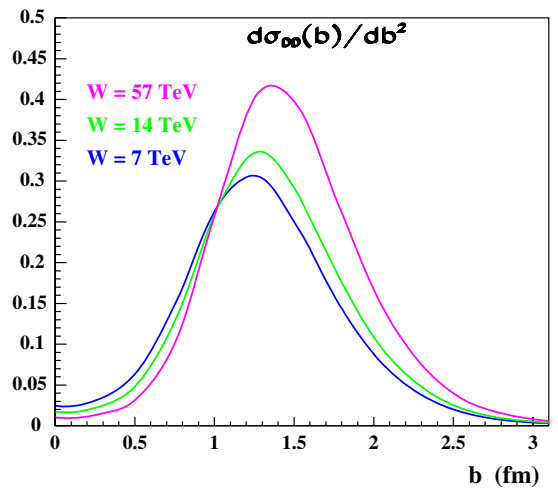

comparison shows that double diffraction is sensitive to the production mechanism and, in particular, to the model of the hadron structure.

We would like to point out the similarity of the distributions of our two channel dipole model (shown in Fig. 7), with those obtained by the Durham group [18], in spite of the difference in the basic premises of the two groups.

\section{Conclusions}

In this paper we demonstrate that the generalization of the CGC/saturation-based model to two channels allows us to describe the experimental data, without having an oscillating behavior for the single diffraction production, and predicting a value for the elastic slope at $W=7 \mathrm{TeV}$ which is smaller than the experimentally measured value. It should be stressed that the values of the phenomenological parameters extracted from the fit satisfy the theoretical expectations. In addition we find new phenomenological small parameters, viz.: $G_{3 \mathbb{P}} / g_{i} \ll 1$ and $m_{i} / m \ll 1$, which allows us to simplify the theoretical formulas.

As has been discussed above, our approximation is valid only for an energy less than (see Eq. (2.1))

$$
Y=\ln \left(s / s_{0}\right) \leq Y_{\max }=\frac{2}{\Delta_{\mathrm{BFKL}}} \ln \left(\frac{1}{\Delta_{\mathrm{BFKL}}^{2}}\right) \approx 29
$$

for $\lambda=0.38$ (see Table 1). Therefore, we can use our approach for energies up to $W=100 \mathrm{TeV}$.

We believe that this paper, together with Ref. [8], lends support to the supposition that a consistent model, based on 
the BFKL Pomeron and the CGC/saturation approach, can be built. We have demonstrated that this model successfully describes data for high energy hadron scattering. In addition, we hope that this paper lends credence to the arguments that the matching with long distance physics (where the confinement of quarks and gluons is essential) can be reached within the CGC/saturation approach; this, without requiring that the soft Pomeron should appear (as a Regge pole).

Acknowledgments We thank our colleagues at Tel Aviv university and UTFSM for encouraging discussions. Our special thanks go to Carlos Contreras, Alex Kovner, and Misha Lublinsky for elucidating discussions on the subject of this paper. This research was supported by the BSF Grant 2012124 and the Fondecyt (Chile) Grant 1140842.

Open Access This article is distributed under the terms of the Creative Commons Attribution 4.0 International License (http://creativecomm ons.org/licenses/by/4.0/), which permits unrestricted use, distribution, and reproduction in any medium, provided you give appropriate credit to the original author(s) and the source, provide a link to the Creative Commons license, and indicate if changes were made.

Funded by SCOAP ${ }^{3}$.

\section{References}

1. Y.V. Kovchegov, E. Levin, Quantum choromodynamics at high energies. in Cambridge Monographs on Particle Physics, Nuclear Physics and Cosmology (Cambridge University Press, Cambridge 2012)

2. E.A. Kuraev, L.N. Lipatov, F.S. Fadin, Sov. Phys. JETP 45, 199 (1977)

3. Ya Ya. Balitsky, L.N. Lipatov, Sov. J. Nucl. Phys. 28, 22 (1978)

4. L.N. Lipatov, Phys. Rep. 286, 131 (1997)

5. L.N. Lipatov, Sov. Phys. JETP 63, 904 (1986). and references therein

6. P.D.B. Collins, in An introduction to Regge theory and high energy physics (Cambridge University Press, Cambridge, 1977)

7. J. Bartels, C. Contreras, G. P. Vacca, arXiv:1411.6670 [hep-th]

8. E. Gotsman, E. Levin, U. Maor, Eur. Phys. J. C 75, 1-18 (2015). arXiv:1408.3811 [hep-ph]

9. A. Donnachie, P.V. Landshoff, Nucl. Phys. B 231, 189 (1984)

10. A. Donnachie, P.V. Landshoff, Phys. Lett. B 296, 227 (1992)

11. A. Donnachie, P.V. Landshoff, Zeit. Phys. C 61, 139 (1994)

12. E. Gotsman, E. Levin, U. Maor, Eur. Phys. J. C 71, 1553 (2011). arXiv: 1010.5323 [hep-ph]

13. E. Gotsman, E. Levin, U. Maor, J.S. Miller, Eur. Phys. J. C 57, 689 (2008). arXiv:0805.2799 [hep-ph]

14. A.B. Kaidalov, M.G. Poghosyan, arXiv:0909.5156 [hep-ph]

15. A.D. Martin, M.G. Ryskin, V.A. Khoze, arXiv:1110.1973 [hep-ph]

16. S. Ostapchenko, Phys. Rev. D 81, 11402 (2010)

17. E. Gotsman, E. Levin, U. Maor, Phys. Lett. B 716, 425 (2012). arXiv:1208.0898 [hep-ph]

18. V.A. Khoze, A.D. Martin, M.G. Ryskin, Eur. Phys. J. C 74, 2756 (2014). arXiv:1312.3851 [hep-ph]

19. Phys. Rev. D 83, 014018 (2011). arXiv:1010.1869 [hep-ph]

20. E. Gotsman, E. Levin, U. Maor, A comprehensive model of soft interactions in the LHC era. arXiv:1403.4531 [hep-ph]

21. Y.V. Kovchegov, E. Levin, Nucl. Phys. B 577, 221 (2000). arXiv:hep-ph/9911523

22. A.H. Mueller, B. Patel, Nucl. Phys. B 425, 471 (1994)

23. A.H. Mueller, G.P. Salam, Nucl. Phys. B 475, 293 (1996). arXiv:hep-ph/9605302
24. G.P. Salam, Nucl. Phys. B 461, 512 (1996)

25. E. Iancu, A.H. Mueller, Nucl. Phys. A 730, 460 (2004). arXiv:hep-ph/0308315

26. E. Iancu, A.H. Mueller, Nucl. Phys. A 730, 494 (2004) arXiv:hep-ph/0309276

27. T. Altinoluk, C. Contreras, A. Kovner, E. Levin, M. Lublinsky, A. Shulkim, Int. J. Mod. Phys. Conf. Ser. 25, 1460025 (2014)

28. T. Altinoluk, N. Armesto, A. Kovner, E. Levin, M. Lublinsky, JHEP 1408, 007 (2014)

29. T. Altinoluk, A. Kovner, E. Levin, M. Lublinsky, JHEP 1404, 075 (2014). arXiv:1401.7431 [hep-ph]

30. T. Altinoluk, C. Contreras, A. Kovner, E. Levin, M. Lublinsky, A. Shulkin, JHEP 1309, 115 (2013)

31. L.V. Gribov, E.M. Levin, M.G. Ryskin, Phys. Rep. 100, 1 (1983)

32. A.H. Mueller, A.I. Shoshi, Nucl. Phys. B 692, 175 (2004). arXiv:hep-ph/0402193

33. I. Balitsky, arXiv:hep-ph/9509348

34. I. Balitsky, Phys. Rev. D 60, 014020 (1999). arXiv:hep-ph/9812311

35. Y.V. Kovchegov, Phys. Rev. D 60, 034008 (1999). arXiv:hep-ph/9901281

36. J. Jalilian-Marian, A. Kovner, A. Leonidov, H. Weigert, Phys. Rev. D 59, 014014 (1999). arXiv:hep-ph/9706377

37. J. Jalilian-Marian, A. Kovner, A. Leonidov, H. Weigert, Nucl. Phys. B 504, 415 (1997). arXiv:hep-ph/9701284

38. J. Jalilian-Marian, A. Kovner, H. Weigert, Phys. Rev. D 59, 014015 (1999). arXiv:hep-ph/9709432

39. A. Kovner, J.G. Milhano, H. Weigert, Phys. Rev. D 62, 114005 (2000). arXiv:hep-ph/0004014

40. E. Iancu, A. Leonidov, L.D. McLerran, Phys. Lett. B 510, 133 (2001). arXiv:hep-ph/0102009

41. E. Iancu, A. Leonidov, L.D. McLerran, Nucl. Phys. A 692, 583 (2001). arXiv:hep-ph/0011241

42. E. Ferreiro, E. Iancu, A. Leonidov, L. McLerran, Nucl. Phys. A 703, 489 (2002). arXiv:hep-ph/0109115

43. H. Weigert, Nucl. Phys. A 703, 823 (2002). arXiv:hep-ph/0004044

44. A.H. Mueller, J. Qiu, Nucl. Phys. B 268, 427 (1986)

45. L. McLerran, R. Venugopalan, Phys. Rev. D 49(2233), 3352 (1994)

46. L. McLerran, R. Venugopalan, Phys. Rev. D 50, 2225 (1994)

47. L. McLerran, R. Venugopalan, Phys. Rev. D 53, 458 (1996)

48. L. McLerran, R. Venugopalan, Phys. Rev. D 59, 09400 (1999)

49. M. Froissart, Phys. Rev. 123, 1053 (1961)

50. A. Martin, Scattering Theory: Unitarity, Analitysity and Crossing. Lecture Notes in Physics (Springer, Berlin, 1969)

51. A.H. Mueller, D.N. Triantafyllopoulos, Nucl. Phys. B 640, 331 (2002). arXiv:hep-ph/0205167

52. D.N. Triantafyllopoulos, Nucl. Phys. B 648, 293 (2003). arXiv:hep-ph/0209121

53. E. Iancu, K. Itakura, L. McLerran, Nucl. Phys. A 708, 327 (2002). arXiv:hep-ph/0203137

54. E. Levin, JHEP 1311, 039 (2013). arXiv:1308.5052 [hep-ph]

55. I. Gradstein, I. Ryzhik, Table of Integrals, Series, and Products, 5th edn. (Academic Press, London, 1994)

56. K.G. Boreskov, A.B. Kaidalov, V.A. Khoze, A.D. Martin, M.G. Ryskin, Eur. Phys. J. C 44, 523 (2005). arXiv:hep-ph/0506211

57. A. Kormilitzin, E. Levin, A. Prygarin, Nucl. Phys. A 813, 1 (2008). arXiv:0807.3413 [hep-ph]

58. E. Levin, A. Prygarin, Eur. Phys. J. C 53, 385 (2008). arXiv:hep-ph/0701178

59. M.L. Good, W.D. Walker, Phys. Rev. 120, 1857 (1960)

60. E. Levin, A.H. Rezaeian, Phys. Rev. D 83, 114001 (2011). arXiv: 1102.2385 [hep-ph]

61. E. Levin, A.H. Rezaeian, A.I.P. Conf, A.I.P. Conf. Proc. 1350, 243 (2011). arXiv:1011.3591 [hep-ph]

62. E. Levin, A.H. Rezaeian, Phys. Rev. D 82, 054003 (2010). arXiv:1007.2430 [hep-ph] 
63. E. Levin, A.H. Rezaeian, Phys. Rev. D 82, 014022 (2010). arXiv:1005.0631 [hep-ph]

64. D. Kharzeev, E. Levin, Phys. Lett. B 523, 79 (2001). http://arxiv. org/abs/nucl-th/0108006. arXiv:nucl-th/0108006

65. J.L. Albacete, N. Armesto, J.G. Milhano, P. Quiroga-Arias, C.A. Salgado, Eur. Phys. J. C 71, 1705 (2011). arXiv:1012.4408 [hep$\mathrm{ph}]$

66. A. Kormilitzin, E. Levin, Nucl. Phys. A 849, 98 (2011). arXiv: 1009.1468 [hep-ph]

67. E. Iancu, J.D. Madrigal, A.H. Mueller, G. Soyez, D.N. Triantafyllopoulos, Resumming double logarithms in the QCD evolution of color dipoles. arXiv:1502.05642 [hep-ph]

68. M.G. Poghosyan, J. Phys. G G 38, 124044 (2011). arXiv: 1109.4510 [hep-ex]

69. ALICE Collaboration, First proton-proton collisions at the LHC as observed with the ALICE detector: measurement of the charged particle pseudorapidity density at $\sqrt{s}=900 \mathrm{GeV}$. arXiv:0911.5430 [hep-ex]
70. G. Aad et al. [ATLAS Collaboration], Nature Commun. 2, 463 (2011). arXiv:1104.0326 [hep-ex]

71. CMS Physics Analysis Summary, Measurement of the inelastic pp cross section at $? \mathrm{~s}=7 \mathrm{TeV}$ with the CMS detector (2011)

72. F. Ferro, TOTEM Collaboration, AIP Conf. Proc. 1350, 172 (2011)

73. G. Antchev et al., TOTEM Collaboration, Europhys. Lett. 96, 21002 (2011)

74. G. Antchev et al. [TOTEM Collaboration], Europhys. Lett. 95, 41001 (2011). arXiv:1110.1385 [hep-ex]

75. G. Antchev et al. [TOTEM Collaboration], Phys. Rev. Lett. 111 (2013) 26, 262001 arXiv:1308.6722 [hep-ex]

76. J. Beringer et al., Particle Data Group, Phys. Rev. D 86, 010001 (2012) 